\title{
Managing preconceived expectations: mental health service users experiences of going home from hospital: a grounded theory study
}

B. KEOGH, ${ }^{1}$ RPN BNS MSc phD, P. CALLAGHAN ${ }^{2}$ RMn bSc mSc phd CPsychol Csci fHeA \& A. HIGGINS ${ }^{3}$ RPN RGN BNS RNT MSC PhD

${ }^{1}$ Assistant Professor, ${ }^{3}$ Professor of Mental Health, School of Nursing and Midwifery, Trinity College Dublin, Dublin, Ireland, and ${ }^{2}$ Professor of Mental Health Nursing, School of Nursing, Midwifery and Physiotherapy, University of Nottingham, Queens Medical Centre, Nottingham, UK

Keywords: discharge planning, grounded theory, mental health, recovery, stigma, service user experiences

Correspondence:

B. Keogh

School of Nursing and Midwifery

Trinity College Dublin

24 D’Olier Street

Dublin 2

Ireland

E-mail:keoghbj@tcd.ie

Accepted for publication: 28 July 2015

doi: 10.1111/jpm.12265

\section{Accessible summary}

What is known on the subject?

- The time of discharge from a mental health hospital can be challenging for mental health service users, with high rates of readmission in the immediate months following discharge.

- Although some research exists that explores service users' perspectives of being discharged, little evidence exists that explores the processes influencing or used by service users' to adapt to the transition from in-patient acute mental health service.

What this papers adds to existing knowledge?

- The findings of this grounded theory study demonstrates the strategies service users used to managed their own, as well as their social audiences, preconceived expectations arising from their new identity as 'psychiatric patients' following their discharge from hospital.

- While there is a move to develop recovery-orientated mental health services, key indicators of recovery-oriented practices were often absent from service users' experiences of service provision.

What are the implications for practice?

- Nurses and other mental health professionals need to recognize their contribution to the architecture of stigma that transcends the physical structures of hospital or ward and are entrenched within attitudes, interactions and practices.

- The findings of this study can provide guidance to those working with service users and help them to understand the complexities of their experiences when using mental health services, which go far beyond the management of their symptoms.

\section{Abstract}

Introduction: Following a period of hospitalization, the transition to home can result in increased vulnerability and a source of stress for mental health service users. Readmission rates have been suggested as one indicator of the success of the transition from hospital to community care. Despite knowledge of some of the factors that impact on service users following discharge, no coherent model or theoretical framework could be located in the literature, which explains or aides an in-depth understanding of the transition from hospital to community for service users. Aim: The aim of this study was to develop a grounded theory that explored service users' experiences of going home from hospital. Method: This qualitative study used grounded theory, and a total of 35 interviews were conducted with 31 service users. Results: The core category was 'Managing Preconceived Expectations', which 
had seven subcategories, describes how the participants were negatively perceived by themselves and others following their admission and discharge from hospital. Implications for Practice: This theory presents the strategies that the participants used to manage this new identity. This theory demonstrates that although there has been a move to adopt recovery-orientated services, key indicators of recovery were often absent for service users being admitted and subsequently discharged.

\section{Introduction and background}

The experience of a mental health problem and admission to a mental health service can be one of the most distressing, frightening and disempowering events in a person's life (Foster 2007, Walsh \& Boyle 2009, Nolan et al. 2011). In addition, coming home from hospital and re-entering the community can also be a challenging experience. Re-adapting to community living is often hampered by the enduring and unpredictable trajectory of mental distress (Beebe 2010). Issues such as social exclusion, loneliness, stigma, housing insecurity and unemployment can negatively impact on service users' recovery, resulting in a high number of people being readmitted (Lemaire \& Mallik 2005, National Economic and Social Forum 2007, Nolan et al. 2011, Parsonage 2013, Siskind et al. 2014). Relapse and subsequent readmission are a major source of distress for service users and their family members and lead to decreased quality of life for service users, acceleration of social disablement, as well as being an economic burden on health services (Chang et al. 2003, Brosnan 2006, Vigod et al. 2013, Siskind et al. 2014). Internationally, there has been a continual drive to locate mental health services in the community. However, despite these changes, research has suggested that adapting to community living following a period of hospitalization is difficult (Lemaire \& Mallik 2005).

Readmission rates have been suggested as one indicator of the success of the transition from hospital to community care (Lien 2002, Durbin et al. 2007, Vigod et al. 2013). Mgutshini (2010) also suggests that readmission to hospital has become a quality indicator and is often interpreted as the failure of the earlier admission. Durbin et al. (2007) in their review on readmission rates suggest that there is evidence to support an association between discharge preparedness and readmission to hospital. This is supported by Nurjannah et al. (2014) who state that effective discharge planning can impact on the frequency of readmissions to hospital as well as individuals' quality of life. Vigod et al. (2013) discuss a framework of interventions that might assist in preventing readmission to hospital: predischarge, postdischarge and bridging interventions. However, despite high rates of psychiatric readmissions, there is a lack of evidence to support them (Vigod et al.
2013). Steffen et al. (2009) completed a systematic review on discharge planning specifically in mental health care. They included 11 studies in their review and found that most of the planning centred on discharge preparation. They suggested that there was some difficulty in determining what constituted discharge and that the heterogeneity of peoples' needs on discharge made discharge planning difficult (Steffen et al. 2009). Dukkers et al. (1999) and Greenberg \& Rosenheck (2005) suggest that in the transition from hospital to community care, service users are often subject to increased vulnerability. Despite this, there is a paucity of research that examines the transition of mental health service users from acute inpatient services to community living.

The difficulties facing people when they are discharged from hospital have often been centred on biomedical- and disease-based models, suggesting that preventing the re-emergence of clinical symptoms (relapse) are key to successful community tenure. Studies here have focused on the efficacy of prescribed medication and its role in preventing relapse primarily in people with a diagnosis of schizophrenia (see Kishimoto et al. 2013 for example). Furthermore studies such as these are often couched in the belief that relapse and readmission to hospital are often inevitable for users of the mental health services. The psychosocial experiences of people leaving hospital and returning to the community have been investigated to some extent using various methodologies (Lorencz 1991, Montgomery \& Johnson 1998, Johnson \& Montgomery 1999, Beebe 2010, Lemaire \& Mallik 2005, Simpson et al. 2014, Nurjannah et al. 2014). The transition of service users into the community in the context of de-institutionalization has also been considered (Machado et al. 2012). Although these studies go some way to explaining individual experiences and the challenges encountered, they fail to provide data that explain the social and psychological transition process for service users discharged from the acute mental health services. In addition, despite awareness of the fact that issues such as vulnerability and community support factors are predictors of readmission (Durbin et al. 2007), no coherent model or theoretical framework could be located in the literature that explains or aides an in-depth understanding of the transition from hospital to community for this group of individuals. This may explain why achieving 
a positive outcome for service users following discharge is, as Simons \& Petch (2002) suggest, something that mental health professionals find challenging. Hence, the aim of this study was to explore service users' experiences perspectives of going home from a psychiatric hospital.

\section{Aim of study}

The aim of the study was to develop a grounded theory (GT) of mental health service users' experiences of going home from the hospital.

\section{Methods}

The study used GT as described by Glaser \& Strauss (1967) and Glaser (1978). Key to GT methodology is the articulation of participant's main concern within a substantive area. The social processes that the participants use to resolve this main concern is the GT that 'emerges' from the data. The theory is generally composed of a core and a related category that have strong explanatory power. Although many variations of the GT method exist (for example Strauss \& Corbin 1998 and Charmaz 2003), the classical method as described by Glaser was used in the development of theory.

\section{Recruitment of participants}

In this study, the participants were recruited from community mental health services and vocational and voluntary organizations in one large urban area in the Republic of Ireland. A total of 35 interviews were conducted with 31 mental health service users. Of the participants, 18 were male and 13 were female; their ages ranged from 25 to 63 years and the average age was 44.5 years. Seven of the participants had one previous admission to hospital; the remainder has between 2 and 33 previous admissions.

\section{Data collection}

Data were collected using individual face-to-face interviews. Early interviews with the participants took an unstructured approach with subsequent interviews becoming more focused as the data collection and analysis progressed. Most of the interviews took place in a mental health day or vocational centre. Two of the interviews were conducted in the participants' home and one was conducted in a public place. In the early interviews, participants were asked about their hospital experiences, their discharge preparation and their experiences when they went home. In keeping with the principles of theoretical sampling, although all areas remained central to subse- quent interviews, additional questions and probes were used to saturate or refute emerging categories.

\section{Data analysis}

Interviews were audio-recorded and transcribed verbatim. Data were collected and analyzed simultaneously using the constant comparative method. Theoretical memos documented the analytic process and emerging theory. Initial open coding generated a voluminous amount of codes that were refined as the data collection process progressed. These were organized into concepts, and connections between concepts were hypothesized by the researchers and then tested through subsequent data collection. Once the main concern was identified and articulated, concepts were organized into categories that explained the social process the participants used to resolve their main concern. The criteria associated with establishing trustworthiness in classic GT research (Glaser 1978) (namely 'fit', 'workability', 'modifiability' and 'relevance') were used in this study.

\section{Ethical issues}

The research was approved by University Ethics Committee and the ethics committees within the data collection sites. All participants received written and verbal information, and those who agreed to participate signed a written consent form. Consent was negotiated throughout the whole research process, and priority was given to participants' well-being. Potential participants were advised not to take part in the study if they felt that talking about their experiences would cause unnecessary upset. In the event that a participant became upset during an interview, a named mental health professional was available to contact should that be required. This facility was not utilized throughout the research study.

\section{Results}

Central to classic GT development is the articulation of the participants' main concern. In this study, the participants' main concern was related to their desire to manage the negative assumptions that their social audience had about them once they were discharged from a psychiatric hospital. The participants in this study were aware that mental distress and psychiatric hospitalization were associated with a number of negative assumptions, as being labelled 'mentally ill' became for them and others, a master status that impacted negatively on all aspects of their lives. The participants resolved this concern with a process conceptualized as 'Managing Preconceived Expectations', which had seven related subcategories: 


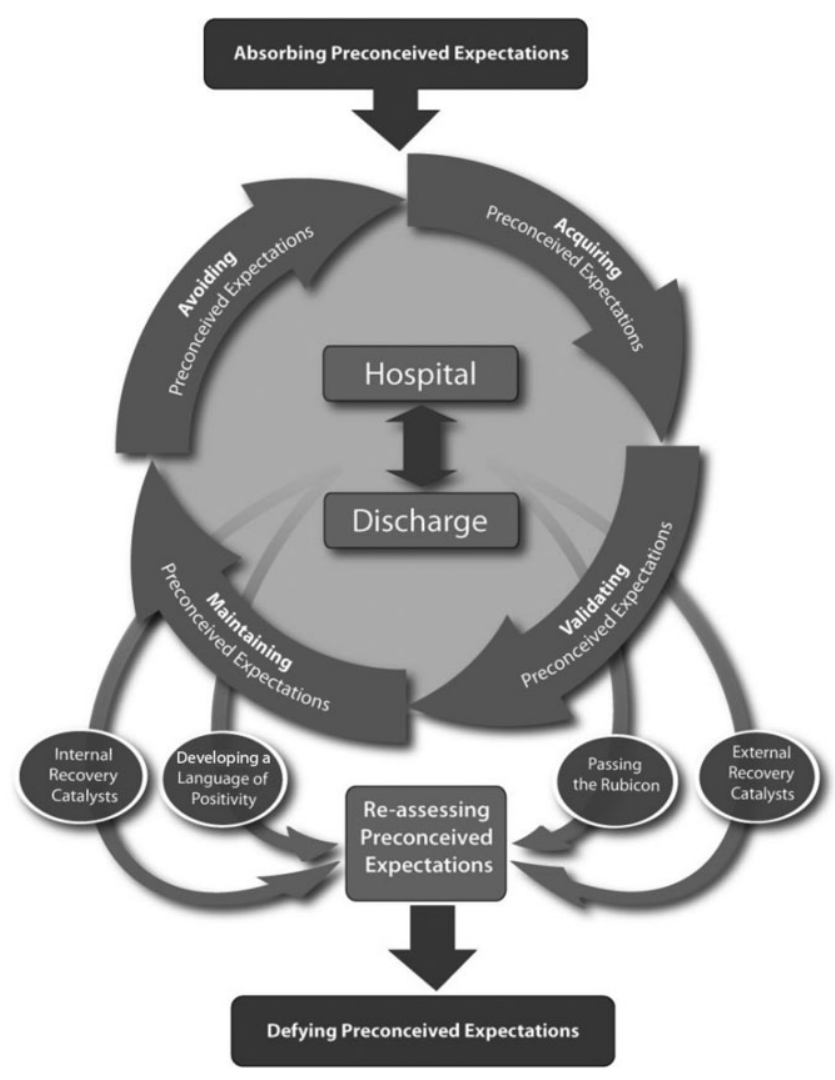

Figure 1

Managing preconceived expectations

1. Absorbing preconceived expectations

2. Acquiring preconceived expectation

3. Validating preconceived expectations

4. Maintaining preconceived expectations

5. Avoiding preconceived expectations

6. Reassessing preconceived expectations

7. Defying preconceived expectations

A diagrammatic view of the categories and their relationship to each other is presented in Fig. 1.

\section{Absorbing preconceived expectations}

'Absorbing Preconceived Expectations' describes how prior to the participants' contact with the mental health services they had already formed a negative conceptualization of mental distress and psychiatric hospitalization. The participants had a perception of mental distress as something that was poorly understood, something that was feared and something to be ashamed of. To a lesser extent, the participants related mental distress with physical illness. In the absence of first-hand knowledge about mental distress or direct contact with people with mental health problems, it was likely that the participants gained this knowledge from the media, or through the discourses they heard about mental distress as they were growing up. The participants' ability to conceptualize mental distress despite the lack of empirical knowledge supports the standpoint that beliefs about mental distress were unconsciously memorized as they were growing up. These beliefs were then reactivated once the participants came into contact with the mental health services (Turner 1982). This lack of knowledge and awareness of mental distress and the influence of the media is highlighted in the following quotation.

Never heard of a psychiatric hospital in my life. Never heard of a psychiatric doctor. Psychiatrists was something I seen on the telly. Never had any information of any of this at all (Female, Interview 30).

\section{Acquiring preconceived expectations}

When the participants were admitted to the hospital, they acquired new and additional beliefs about mental distress. This occurred primarily through their contact with the mental health professionals that they encountered in the hospital. In addition, the conceptualizations of mental distress that they had already formed were subtlety reinforced. When they looked for explanations as to why they were experiencing particular feelings, they were given explanations using a biomedical discourse that fostered the belief that they were sick and that mental distress was a lifelong, recurrent illness where the prospect of recovery was slim. Practices such as having to wear nightwear or pyjamas and not being allowed to leave the ward heightened the sense that they were sick and needed to be cared for. Furthermore, the mental health professionals that they encountered discouraged the participants from openly discussing their mental health problems when they were discharged from the hospital. This heightened the belief that mental distress was something to be ashamed of and something that should remain hidden from others.

\section{Validating preconceived expectations}

'Validating Preconceived Expectations' is the third category in this theory and describes how the conceptualizations that the participants had absorbed and acquired about mental distress were further validated when they were discharged from the hospital. The participants in this study used a custodial language to describe their discharge and talked about 'being released from hospital' rather than being discharged. Although the participants anxiously sought their discharge, they were fearful about going home. This was articulated as 'feeling different'. Despite their anxiety about being discharged, none of the participants had any specific preparation or say in the decision about going home. 
Well they [the doctors and nurses] tell you when you're ready to go home, you know like if you wanted to discharge yourself, anytime you can do it, but I used to just wait, they'd tell you when you're ready (Female, Interview 1).

Once discharged, the encounters the participants had with their social audience validated and reinforced their perceptions of mental distress as lifelong and life limiting. Furthermore their encounters with mental health professionals in the community furthered their conception of mental distress as illness while also reinforcing their lack of involvement and choice in treatment regimes. Encounters with their family and friends also augmented the belief that mental distress should not be openly discussed and that it was something to be ashamed of:

I think it was, I don't know, I don't know, it was like being really ashamed of being mentally ill because and guilty for putting my family through, you know through a tough time you know (Female, Interview 27).

\section{Maintaining preconceived expectations}

Following discharge, a number of additional experiences maintained the negative assumptions that the participants had developed about themselves as people who had a mental health problem and who required admission to a mental health service. The processes described mainly deal with the broad experience of mental distress-based stigma, which continually reinforced the participants' beliefs about the nature of their experience and about themselves as discredited individuals. The stigma experiences were based on preconceived assumptions about mental distress and people who use the mental health services. This category also demonstrates how the participants internalized the negative assumptions associated with mental distress and how these impacted on their self-esteem and their perceptions of themselves. Participants described being avoided or shunned by people and felt that their social audience did not understand them or their experiences. The participants talked about being inadequate and feeling hopeless, and in some cases, their mental distress was exacerbated because of their negative self-belief. Furthermore encounters with myths and misconceptions about mental distress, for example the belief that people with mental illness are dangerous, continually aided their negative self-perceptions and strengthened their negative conceptualizations of mental distress.

Everything changed after I got sick really, I lost a lot of friends, like I did . . . we all hung out together and I was kind of like nudged out of the group, you know and like they'd be going out and I was kind of like nudged out of the group, you know and like they'd be going out like and I wasn't invited and you know they were a bit nasty now and I was bullied by them (Female, Interview 26).

\section{Avoiding preconceived expectations}

'Avoiding Preconceived Expectations' describes the many processes that the participants used to avoid or minimize the stereotypes associated with being a psychiatric patient. First, they socially disengaged by distancing themselves from social relationships that they had prior to hospitalization. This was often in response to embarrassment or anxiety about having to explain their absence or explain what they were doing now to people they knew. This often meant that the participants' friends and social network drifted away and lost contact. The participants also used cautious disclosure to manage their fear of being negatively stereotyped. This involved a range of processes from nondisclosure through to the creation of an alternative biography where the participants fabricated a new history to avoid revealing their history of mental distress. In most instances, the participants used selective disclosure, which involved either disclosing their experiences to a limited number of people or admitting that they had a diagnosis that they thought sounded less 'severe' (e.g. saying they had depression rather than schizophrenia). The participants often made allowances for their exclusions and stigmatization and suggested that it occurred because of ignorance about mental distress. In addition, the drifting away of friends and other social contacts was often reported as a natural progression of events, which the participants were unable to achieve because of their mental health problem. Prior to openly discussing their mental distress, the participants often gauged the reactions of their audience to see if their revelations would be positively received or took time to get to know the person before disclosing as in the following quotation:

But then again meeting new people, like for instance meeting new girls or meeting new friends and stuff like that that I wouldn't have known before, I think it's, I'd like to explain to them meself [myself] the ins and outs of what I went through at some stage, like slowly explain to them but I wouldn't be able to jump into it and tell them straightaway kind of thing (Male, Interview 16).

\section{Reassessing preconceived expectations}

'Reassessing Preconceived Expectations' illuminates the range of psychological and social occurrences, which assisted the participants to reassess their experiences and to reframe them in the light of a greater understanding of 
mental health and recovery. The participants' mental health problems and their experiences, which had become a 'master status', was challenged by some participants through self-reappraisal and through personal development. 'Reassessing Preconceived Expectations' is precipitated by a major turning point in the participants' lives. Although this was a major turning point, it did not necessarily mean that it was precipitated by a major event. For the participants who reached this point, it was generally a gradual dawning or realization that they could no longer continue to live their lives worried about what other people thought of them. In addition, the participants described a range of internal and external recovery catalysts that assisted the participants to reconceptualize and recontextualize their experiences with mental distress and the mental health services. This allowed the participants to view themselves and their experiences in a more positive light. In tangent with these processes, the participants began to develop a language of positivity, which provided them with a greater comprehension of their experiences. This often involved educating themselves about mental health issues that affected them and building a supportive network of individuals including other mental health service users. The range of internal and external recovery catalysts used by the participants provided the participants with motivation, hope and a desire to remain on a positive recovery trajectory:

Well I couldn't answer that [what made him think about his mental distress differently] really because I just sort of said one day there's thousands like me, you know what I mean. There's thousands out there that have schizophrenia, you know and I said I'm not the only one you know. It's knowing that your life has to go on (Male, Interview 21).

\section{Defying preconceived expectations}

'Defying Preconceived Expectations' describes how some of the participants moved along a recovery trajectory to this final point in managing their concerns about other peoples' preconceived expectations about them following their discharge from hospital. Not all of the participants in this study managed to reach this particular stage in the process; however, many did speak of the recovery catalysts described earlier. For some, the recovery catalysts provoked a re-evaluation of their position as a 'psychiatric patient' and challenged the persisting and pervasive view that people with mental health problems are no longer active or contributing members of society. In addition, they challenged the notion that mental health problems are chronic and debilitating illnesses or disorders. This category emerged in direct response to a desire to defy these negative assumptions. For the participants in this study, casting off the preconceived shackles of what it meant to have a mental health problem was important to their selfdevelopment as an individual and for some it replaced their earlier avoidance or denial of their mental health status. The participants defied preconceived expectations in two ways. First, by 'becoming a success', participants reported they defied the expectation that they were unable to have successful outcomes following their 'illness' and hospitalization (e.g. getting a job). Second, by 'maintaining mental health', they defied the expectation that their illness was lifelong and unremitting and that they are always close to 'having a nervous breakdown' or being admitted to hospital again:

I would see myself as having had schizophrenia, not having it, .... I think do I still have to call myself a schizophrenic for the rest of my life because I'm not a schizophrenic, I'm someone that had experience with schizophrenia (Male, Interview 16).

\section{Discussion}

The aim of this study was to develop a GT of the participants' experiences of going home from the hospital. The substantive theory that emerged from the data provides a conceptual framework that describes the participants' experiences of stigma following admission to and subsequent discharge from hospital. In examining these experiences, the negative assumptions that exist about people who use the mental health services were influential prior to their admission to hospital, during their admission and once they were discharged. Central to this experience of stigma were the negative attitudes demonstrated by mental health professionals, the participants' families and their friends. Furthermore, the negative attitudes to which they were exposed fuelled participants' negative impression of themselves once they came into contact with the mental health services. The 'Managing Preconceived Expectations' theory demonstrates that despite the existence of mental distress-based stigma, people with mental health problems can manage the negative assumptions that are held about them through avoidance, although this is not ideal. In addition, the theory demonstrates that for some of the participants in this study, they were able to defy these negative assumptions. However, this movement along a recovery trajectory is contingent on a number of inter- and intrapersonal factors that have been described in this theory as recovery catalysts. The theory also demonstrates the effectiveness of recovery-orientated strategies such as education, self-management and self-responsibility and the limitations of traditional conceptualizations of mental distress. 
Traditionally, the concept of recovery in mental health has been judged primarily on the absence of psychiatric symptoms and a reduction in the number or frequency of hospital admissions (Durbin et al. 2007). More recently, there has been an emerging desire by mental health policy developers and service providers to reconceptualize recovery in tandem with Anthony's (1993) definition. He suggests that recovery is more than just the reduction or remission of symptoms but is wider in terms of the individuals' ability to lead a life that is full and complete in the presence or absence of 'symptoms' (Anthony 1993). Despite the emphasis on recovery within international mental health policy, it has been difficult to capture its essence in terms of service orientation and delivery (Slade et al. 2014). While there is a growing body of literature to support recovery-orientated practices for mental health professionals, there is a lack of clarity about how this evidence can be translated into practice (Lakeman 2010, Slade et al. 2012). The 'Managing Preconceived Expectations' aids our understanding of the processes that occur during hospitalization and subsequent discharge and highlight the factors that negatively and positively influence recovery during this time.

The participants' concerns in this study centred on how other people perceived them once they were labelled as 'mentally ill'. Consequently, their recovery involved reconciliation with their discredited self-concept rather than their diagnosed 'illness'. Soundy et al. (2015) suggest that it is important to understand how essential the concept of identity is for people in terms of their personal recovery. In this study, the experience of being diagnosed (labelled) and admitted to hospital had a far-reaching negative impact that altered the participants' sense of self and their perception of themselves as valid individuals. For the participants in this study, their experience as 'psychiatric patients' became a 'master status' consequently overriding their other identities (Becker 1963, Erikson 1966). The negative impact that psychiatric hospitalization can have on individuals needs to be acknowledged by mental health professionals. In order to support personal recovery for the individuals that are admitted to the hospital, mental health professionals need to be cognisant of and sensitive to the individuals' pre- and posthospital experiences and their impact on identity (Le Boutillier et al. 2011, Soundy et al. 2015). One of the challenges facing the implementation of recovery-orientated practices is the belief that recovery as a concept is not applicable to people who are acutely unwell or that recovery only begins when individuals are discharged (Slade et al. 2014). These and other myths associated with recovery need to be addressed and challenged if models of care within acute services are to change. In this study, the impact of being diagnosed as 'mentally ill' and the stigma associated with being admitted to the hospital were not often considered by the mental health professionals. When it was acknowledged by professionals, it was to foster and encourage cautious disclosure, which could be seen as reinforcing stigma. Facilitating participants to develop strategies that prepared them to challenge societal stigma and discrimination may have been more beneficial (Le Boutillier et al. 2011). In addition, strategies that help individuals to feel better about themselves and to be happy with the person that they are, important recovery indicators (Law \& Morrison 2014), could have facilitated better outcomes on discharge.

In addition, service users' experiences of mental distressbased stigma interacted with and augmented their sense of detachment and social isolation. In that sense, it is imperative that service users are given an opportunity to explore and reflect on their experiences and facilitated to work through any negative self-beliefs that they might have. Although a number of structured programmes that tackle self-stigma have been evaluated positively in the literature (for example MacInnes \& Lewis 2008 and Knight et al. 2006), in the absence of such programmes, using some of the general principles within a person-centred recoveryorientated care plan may allow service users to challenge negative stigmatizing actions as illegitimate (MacInnes \& Lewis 2008). Other interventions that enhance esteem for service users such as fostering a sense of personal empowerment (Corrigan \& Calabrese, 2006) seek to return the locus of control to the service user and in that sense mirror the components of a recovery-orientated approach. Empowerment strategies concentrate on the service users' strengths rather than their weaknesses or limitations, and look at the wider impact of mental distress rather than focusing on symptomatology. Consequently, issues such as employment, social relationships and housing are also foci for intervention (Corrigan \& Calabrese 2006). Educational strategies for people who use the mental health services that concentrate on counteracting the myths that are often held about people with mental health problems are also crucial in helping service users challenge the negative beliefs that they may hold about themselves (Berge \& Ranney 2005). Finally, assisting service users with disclosure issues is also important and needs to be considered as part of recovery-orientated approaches.

\section{Implications for mental health nursing}

Mental health nurses and other professionals need to be aware of the impact that psychiatric hospitalization has on service users sense of self. In addition, they need to acknowledge that stigma continues to be a problem for people with mental health problems and that service users 
need to be prepared to address some of these issues when they are discharged from the hospital. Adopting recovery orientated approaches that focus on the wider impact of mental distress on service users will assist in understanding and addressing some of these issues.

\section{Conclusion}

The 'Managing Preconceived Expectations' theory aids our understanding of the participants' experiences by presenting a conceptual framework based on their qualitative descriptions of hospitalization and subsequent discharge home. In doing so, it offers a greater understanding of the factors that inhibit or facilitate recovery for individuals who are struggling to make meaning from their experience of mental distress. Although this theory was written in the context of hospital discharge, it is the participants' conceptualizations of mental distress prior to admission and their hospital experiences that firmly shape their interactions with the social world postdischarge. The context of the study (i.e. discharge from hospital) supports the argument that psychiatric hospitalization is stigmatizing and that mental health services should be located in the community. Despite the relocation of most acute psychiatric wards to general hospitals, the findings from this study suggest that psychiatric wards still conjure up negative images that influence the attitudes and behaviours of the general public and people who use the mental health services. As it is likely that psychiatric hospitalization will remain a prominent feature of the mental health services, this presents as a major challenge to those committed to recovery-orientated approaches. The experiences of the participants in this study illuminate the subtle yet complex processes that contribute to their discredited identity, which is then often confirmed by their social audience once they are discharged from hospital. Nurses and other mental health professionals need to recognize their contribution to the architecture of stigma that transcends the physical structures of hospital or ward and are entrenched within attitudes, interactions and practices. The theory presented here can provide guidance to those working with service users and help them to understand the complexities of the service users' experiences when using the mental health services, which go far beyond the management of their symptoms.

\section{Acknowledgments}

The authors would like to thank the participants for sharing their experiences. In addition we would like to thank the many gatekeepers who provided assistance with access and recruitment. This research benefited from a one year research fellowship from the Health Research Board, Ireland.

\section{References}

Anthony W.A. (1993) Recovery from mental illness: the guiding vision of the mental health service system in the 1990s. Psychosocial Rehabilitation Journal 16, 11-23.

Becker H. (1963) Outsiders: Studies in the Sociology of Deviance. The Free Press, New York.

Beebe L. (2010) What community living problems do persons with schizophrenia report during periods of stability? Perspectives in Psychiatric Care 46, 48-55.

Berge M. \& Ranney M. (2005) Self-esteem and stigma among persons with schizophrenia: implications for mental health. Care Management Journals 6, 139-144.

Brosnan L. (2006) What Part of the Picture? Western Alliance for Mental Health, Galway.

Chang S., Chiu Y. \& Liou I. (2003) Risks for unplanned hospital re-admission in a teaching hospital in southern Taiwan. International Journal of Nursing Practice 9, 389-395.

Charmaz K. (2003) Grounded Theory: Objectivist and constructivist methods. In: Strategies of
Qualitative Inquiry (eds Denzin, N. \& Lincon, Y.), pp. 249-291. Sage Publications, London.

Corrigan P. \& Calabrese J. (2006) Strategies for assessing and diminishing self-stigma. In: On the Stigma of Mental Illness: Practical Strategies for Research and Social Change (ed Corrigan, P.), pp. 239-256. American Psychological Association, Washington, DC.

Dukkers D., Ros W. \& Berns M. (1999) Transition of care: an evaluation of the role of the discharge liaison nurse in the Netherlands. Journal of Advanced Nursing 30, 1186-1194.

Durbin J., Lin E., Layne C., et al. (2007) Is readmission a valid indicator of the quality of inpatient psychiatric care. Journal of Behavioural Health Services and Research 34, 137-2007.

Erikson K. (1966) Wayward Puritans: A Study in the Sociology of Deviance. John Wiley \& Sons, New York.

Foster J. (2007) Journeys Through Mental Illness: Client Experiences and Understandings of Mental Distress. Palgrave Macmillan, Hampshire.

Glaser B. (1978) Theoretical Sensitivity. The Sociology Press, San Francisco, CA.
Glaser B. \& Strauss A. (1967) The Discovery of Grounded Theory. Aldine De Gruyter, New York.

Greenberg G. \& Rosenheck R. (2005) Continuity of care and clinical outcomes in a national health system. Psychiatric Services 56, 427433.

Johnson B. \& Montgomery P. (1999) Chronically mentally ill individuals re-entering the community after hospitalisation. Phase II: the urban experience. Journal of Psychiatric and Mental Health Nursing 6, 445-451.

Kishimoto T., Agarwal V., Kishi T., et al. (2013) Relapse prevention in schizophrenia: a systematic review and meta-analysis of secondgeneration antipsychotics versus first-generation antipsychotics. Molecular Psychiatry 18, 53-66.

Knight M., Wykes T. \& Hayward P. (2006) Group treatment of perceived stigma and self-esteem in schizophrenia: a waiting list trial of efficacy. Behavioural and Cognitive Psychotherapy 34, 305-318.

Lakeman R. (2010) Mental health recovery competencies for mental health workers: 
a Delphi study. Journal of Mental Health 19, 55-67.

Law H. \& Morrison A. (2014) Recovery in psychosis: a Delphi study with experts by experience. Schizophrenia Bulletin. doi: 10.1093/ schbul/sbu047.

Le Boutillier C., Leamy M., Bird V., et al. (2011) What does recovery mean in practice: a qualitative analysis of international recovery orientated practice guidance. Psychiatric Services 62, 1470-1476.

Lemaire G. \& Mallik K. (2005) Barriers to community integration for participants in community based psychiatric rehabilitation. Archives of Psychiatric Nursing 19, 125-132.

Lien L. (2002) Are re-admission rates influenced by how psychiatric services are organised? Nordic Journal of Psychiatry 56, 23-28.

Lorencz B. (1991) Becoming ordinary: leaving the psychiatric hospital. In: The Illness Experience: Dimensions of Suffering (eds Morse, J.M. \& Johnson, J.L.), pp. 140-200. Sage Publications, London.

Machado V., Leonidas C., Santos M., et al. (2012) Psychiatric readmission: an integrative review of the literature. International Nursing Review 59, 447-457.

MacInnes D. \& Lewis M. (2008) The evaluation of a short group programme to reduce self-stigma in people with serious and enduring mental health problems. Journal of Psychiatric \& Mental Health Nursing 15, 59-65.

Mgutshini T. (2010) Risk factors for psychiatric re-hospitalisation: an exploration. International Journal of Mental Health Nursing 19, 257-267.
Montgomery P. \& Johnson B. (1998) Chronically mentally ill individuals re-entering the community after hospitalisation. Journal of Psychiatric and Mental Health Nursing 5, 497503.

National Economic and Social Forum (2007) Mental health and social inclusion. NESDO, Dublin.

Nolan P., Bradley E. \& Brimblecombe N. (2011) Disengaging from acute inpatient psychiatric care: a description of service users' experiences and views. Journal of Psychiatric and Mental Health Nursing 18, 359-367.

Nurjannah I., Mills J., Usher K., et al. (2014) Discharge planning in mental health care: an integrative review of the literature. Journal of Clinical Nursing 23, 1175-1185.

Parsonage M. (2013) Welfare advice for people who use mental health services. Centre for Mental Health, London.

Simons L. \& Petch A. (2002) Needs assessment and discharge: a Scottish perspective. Journal of Psychiatric and Mental Health Nursing 9, 435445 .

Simpson A., Flood C., Rowe J., et al. (2014) Results of a pilot randomised controlled trial to measure the clinical and cost effectiveness of peer support in increasing hope and quality of life in mental health patients discharged from hospital in the UK. BMC Psychiatry 14, 30. doi: 10.1186/1471-244x-14-30.

Siskind D., Harris M., Diminic S., et al. (2014) Predictors of mental health-related acute service utilisation and treatment costs in the 12 months following an acute psychiatric admission.
The Australian and New Zealand Journal of Psychiatry 48, 1048-1058.

Slade M., Adams N. \& O'Hagan M. (2012) Recovery: past progress and future challenges. International Review of Psychiatry 24, 1-4.

Slade M., Amering M., Farkas M., et al. (2014) Uses and abuses of recovery: implementing recovery orientated practices in mental health systems. World Psychiatry 13, 12-20.

Soundy A., Stubbs B., Roskell C., et al. (2015) Identifying the facilitators and processes which influence recovery in individuals with schizophrenia: a systematic review and thematic analysis. Journal of Mental Health 24, 103-110.

Steffen S., Kosters M., Becker T., et al. (2009) Discharge planning in mental health care: a systematic review of the recent literature. Acta Psychiatrica Scandinavica 120, 1-9.

Strauss A. \& Corbin J. (1998) Basics of Qualitative Research: Techniques and Procedures for Developing Grounded Theory, 2nd edn. Sage Publications, Thousand Oaks, CA.

Turner J. (1982) Towards a cognitive redefinition of the social group. In: Social Identity and Intergroup Relations (ed Tajfel, H.), pp. 15-40. Cambridge University Press, Cambridge.

Vigod S., Kurdyak P., Dennis C., et al. (2013) Transitional interventions to reduce early psychiatric readmissions in adults: systematic review. British Journal of Psychiatry 202, 187-194.

Walsh J. \& Boyle J. (2009) Improving acute psychiatric hospital services according to inpatient experiences: a user-led piece of research as a means to empowerment. Issues in Mental Health Nursing 30, 31-38. 